\title{
EXPRESSION OF $\beta$-CATENIN AND E-CADHERIN IN BREAST DUCTAL CARCINOMA IN SITU AND THEIR ASSOCIATION WITH SURVIVAL: FOLLOW UP OF 9.0 YEARS
}

Giuliano M. Duarte, Helena Slongo', Marcelo L. Montenegro', Fernando Tocchet', Juliana Espinola', Fernando Augusto Soares², Priscila Marshall1', Geisilene R. P. Silva'

${ }^{1}$ Woman' Hospital Prof. Dr. José Aristodemo Pinotti, Universidade Estadual de Campinas - Campinas (SP), Brazil. ${ }^{2}$ AC Camargo Cancer Center - São Paulo (SP), Brazil.

Introduction: Tumor invasion and metastatic status still lead the poor prognostic in breast cancer. $\beta$-catenin and e-cadherin are components of cadherin-based cell-to-cell adhesion and also an important intermediate in several signal transduction pathways, including the Wnt pathway. Both are related to invasion, progression, and recurrence of cancer, but there are only few studies regarding in ductal carcinoma in situ (DCIS). The aim of study is to evaluate $\beta$-catenin and e-cadherin expression in pure DCIS and correlate these expressions with immunohistochemistry pattern, disease free survival and local recurrence. Methods: A retrospective cohort study was performed using the records from 1999 to 2009 in our Institution. The medical files of all patients with pure DCIS were reviewed and clinical, treatment and local of recurrence data were obtained. A tissue microarray paraffin block (TMA) was constructed from all biopsies. The TMA was submitted to immunohistochemical staining for $\beta$-catenin, e-cadherin, claudin- 4 , estrogen receptor, progesterone receptor, HER-2 and Ki-67. $\beta$-catenin and e-cadherin were categorized on low or high expression depending on score intensity and quantity. Results: It was included 137 patients with pure DCIS and 68 could have TMA analyzed for $\beta$-catenin and e-cadherin. The mean diagnostic age was $52.31 \pm 11.12$ years. Total local recurrence rate was $11.76 \%$ (50.0\% were invasive carcinoma) after median follow up of 9.06 years. High expression of $\beta$-catenin and e-cadherin were $71.93 \%$, and $87.5 \%$, respectively. High expression of $\beta$-catenin was related to high expression of claudin-4 $(p=0.017)$, e-cadherin $(p=0.041)$, positive estrogen receptor $(p=000.8)$, and positive progesterone receptor $(p=0.048)$, but not to HER-2 and Ki-67. High expression of e-cadherin was associated with high expression of claudin-4 ( $\mathrm{p}=0.005)$ and $\beta$-catenin ( $p=0.017)$, but not others variables. There was no difference in local disease free survival between high and low expression of $\beta$-catenin $(p=0.955)$ and e-cadherin $(p=0.890)$. Conclusion: High expression of $\beta$-catenin and e-cadherin was more frequent than low expression in DCIS. Although, $\beta$-catenin was related to others adhesion components (as claudin and E-cadherin), and hormonal receptors (that are known prognostic factors), there was no association of local recurrence or disease free survival. E-cadherin expression was also not correlated with disease free survival. 